\title{
RESEÑA \\ COLOMBIA 4.0 \\ RETOS Y PERSPECTIVAS \\ SOBRE EL DESARROLLO \\ DE LA CUARTA REVOLUCIÓN INDUSTRIAL
}

Katty Dayanna Valencia Wenndy Pedraza

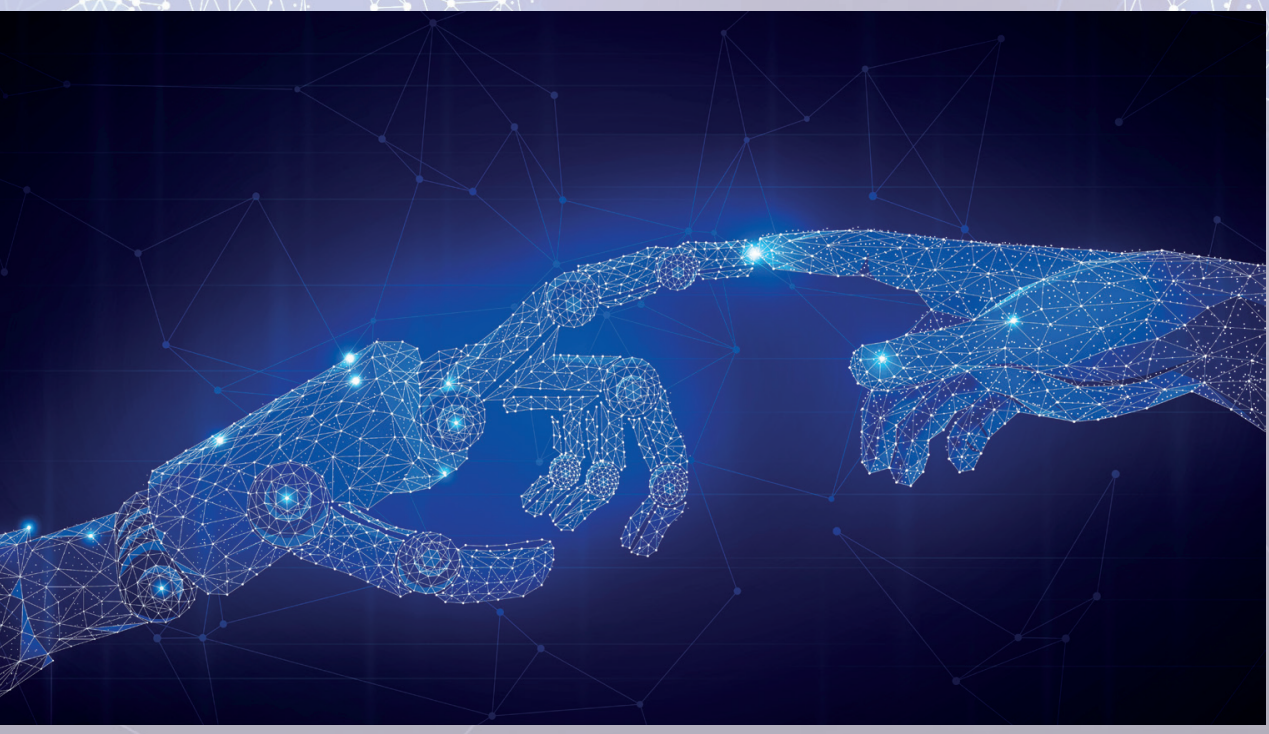





\title{
RESEÑA
}

\section{COLOMBIA 4.0}

\section{RETOS Y PERSPECTIVAS SOBRE EL DESARROLLO DE LA CUARTA REVOLUCIÓN INDUSTRIAL}

\author{
Eduardo Andrés Perafán del Campo, laura Catalina Guerrero \\ Escobar, Catalina Miranda Aguirre, Alejandro León Quiroga, \\ Sebastián Polo Alvis, Geraldine Herrera, Jairo Becerra, \\ Jeice Hernández, Luis Fernando León Quiroga, \\ Marco Emilio Sánchez Acevedo, Natalia Rocío Franco Fuquen, \\ Paula Andrea Pérez Gómez
}

La cuarta revolución industrial (4RI) es un tema altamente abordado en la actualidad, debido a la pertinencia de sus implicaciones en materia de tecnologías e inteligencia artificial. En muchos espacios, se tiene como tema agotado, pero no es así en Colombia, donde se ha desarrollado de forma tardía. Por ello, en este libro se contextualizan las incidencias de dicha revolución en Colombia, teniendo como antecedente principal el lanzamiento del Centro para la Cuarta Revolución Industrial en Medellín, fundado en 2009 por el Ministerio de Comercio, Industria y Turismo. Fue este mismo Ministerio el que introdujo al país a la red de centros asociados para la 4RI, lo que generó consecuencias en todas las esferas de la sociedad y desafíos que se exponen en el desarrollo del libro. Dichos desafíos van desde lo socioeconómico, la protección de datos personales en el uso de tecnologías, la transformación del concepto de trabajo y derechos laborales, la seguridad jurídica, la implementación de la inteligencia artificial en la dinámica de la justicia, hasta las áreas del diseño y las artes. El libro hace una recopilación multidisciplinar, y a pesar de utilizarse el lenguaje técnico de cada materia, ofrece al lector un pliego de definiciones previas que dan claridad y lo aterrizan sobre las implicaciones del concepto de revolución industrial y el fenómeno que es en sí mismo. 
Finalmente, termina con una serie de recomendaciones y reflexiones de los autores sobre el desarrollo y la realidad que desde ya implica la 4RI en Colombia.

El texto se encuentra dividido en seis capítulos, los cuales se esbozan por temáticas generales; estos, a su vez, se subdividen por aspectos específicos que engloba cada temática, lo que le facilita al lector su comprensión.

En el primer capítulo se ofrece una visión histórica como antesala de los temas por desarrollar; en ella se resumen las tres revoluciones industriales, teniendo como punto de partida el siglo XVIII. Se hace énfasis en las implicaciones que tienen estos cambios y avances científicos en los modelos económicos y sociales, y se explican a grandes rasgos los cambios más significativos, además de algunos inventos que transformaron la historia de la humanidad; por ejemplo, la implementación de un nuevo sistema postal, o la nueva perspectiva de la producción desde el fordismo y el taylorismo. Posterior a ello, se hace un repaso por el concepto de "globalización" para entender cómo los mercados, su expansión y la apertura económica entre Estados hacen de este un factor determinante en cada una de las revoluciones industriales.

En la sección final de este capítulo se exponen las características principales y algunas de las implicaciones de la 4RI para América Latina y Colombia, que tienen como consecuencia beneficios y avances en el desarrollo social, político y económico (especialmente en Colombia, que obtuvo la segunda fase de centros para la 4RI), teniendo en cuenta que el factor común en esta región es que se trata de países en vías de desarrollo que aún no cuentan con ciertos mecanismos para responder a dichos cambios.

Seguidamente, en el segundo capítulo se establecen los ocho desafíos que tiene Colombia para poder llevar a cabo la 4RI. Tales desafíos se desprenden de necesidades que actualmente se encuentran sin resolver en la sociedad colombiana, y que, de no trabajarse en ellas, podrían generar un impacto negativo a raíz de la revolución. Inicialmente, se desarrolla cada reto sustentado en fuentes cualitativas y cuantitativas, y en una segunda parte de este capítulo se dan unas recomendaciones para asumir cada desafío. Estas representan una posible solución que se puede dar desde el aspecto político, de distribución de recursos, estrategias de educación, entre otros.

Los restos socioeconómicos para Colombia a raíz de la 4RI, según el texto, son:

- Inclusión de la mujer.

- Transparencia institucional y libertad cibernética. 
- Desarrollo rural y territorialidad.

- Educación.

- Gobernanza ágil.

- Privatización de la ciencia y su relación con la inequidad social.

- Humanidades y ética.

- E-commerce.

En el tercer capítulo se estudian los posibles conflictos que surgirán en el plano laboral colombiano tras la 4RI.

Inicialmente se refiere a los postulados marxistas que surgieron con la primera revolución industrial, para hacer un paralelo con la época actual y demostrar la obsolescencia de algunos aspectos. Luego se hace un análisis teniendo en cuenta que uno de los principales efectos de cualquier revolución industrial es la sistematización de labores que en algún momento fueron desarrolladas por el ser humano. Ello en el contexto de desempleo e informalidad en las fuentes laborales de Colombia, crea un escenario que dista del ideal de la 4RI, ya que, para poder implementar políticas y/o estrategias que contrarresten los posibles efectos negativos, inicialmente se debe dar tratamiento oportuno a los problemas actuales e iniciar la inclusión del sector rural; esto es muy importante, pues las cifras de tales problemáticas son mayores respecto a las del sector urbano.

Luego, se abre paso al sector jurídico, que sin lugar a dudas es determinante para enfrentar los cambios estructurales y sociales que se puedan presentar en cualquier Estado, ya que es la herramienta para regular las relaciones e interacciones de la vida en sociedad. La 4RI, desde luego, implica cambios en los sistemas jurídicos, que vinculados con los avances tecnológicos podrían representar beneficios de incalculable valor para el país, pero que, de igual manera, representan cierta incertidumbre respecto a la seguridad y a la estabilidad de los datos de los ciudadanos y las decisiones judiciales que se adopten.

En este apartado del libro, los autores se enfocan inicialmente en describir conceptos y analizar la situación actual del sistema jurídico en Colombia, con la finalidad de entender qué tan idóneo es el escenario para recibir e incorporar estas variaciones (por ejemplo, las de tipo tecnológico) dentro de un sistema que pueda reaccionar orgánicamente sin limitar los derechos de los ciudadanos. Tal análisis se realiza de manera profunda, tocando temas que rozan con los factores económicos, la producción normativa del país, la vinculatoriedad de dichas normas y las estructuras tanto institucionales como políticas. 
En el quinto capítulo se desarrollan algunos de los conceptos que caracterizan, diferencian y marcan innovación en la 4RI respecto a otras revoluciones industriales, como son los de big data e inteligencia artificial. El primero se encarga de la recopilación de información a gran escala y, el segundo, de simular mediante algoritmos procesos complejos. Estos conceptos, si bien representan ahorro en tiempo y recursos para las economías y agilidad para combatir problemáticas sociales, también generan expectativas e incertidumbre en factores como el derecho y la seguridad de los datos sensibles o de tratamiento especial; además, representan un panorama que tiende un poco a fomentar la falta de innovación y el facilismo en la mente humana, y llega nuevamente a resaltar el reemplazo del hombre por la tecnología.

En relación con lo anterior, en cuanto al derecho, en este capítulo se aborda la necesidad de reevaluar conceptos jurídicos base sobre los cuales se construyen los atributos de la personalidad. En torno a la creación de inteligencia artificial semejante a la humana (los robots), los autores realizan un análisis sobre la revaloración de estos conceptos que demuestran la completa transformación que se aproxima con la 4RI.

En este capítulo, desde la perspectiva de la 4RI, se presentan los cambios que se generan en factores indispensables para el desarrollo de la sociedad, como lo son el arte y la cultura. Así, se hace énfasis en los nuevos y predominantes sistemas de comunicación, que se basan en el contenido visual como apoyo al mercadeo y las profesiones relacionadas con el diseño. Se marca la relevancia de las nuevas herramientas que se nombran en capítulos anteriores, por ejemplo, el big data, y la participación de artistas en nuevas metodologías de desarrollo de contenido audiovisual, por medio de la realidad aumentada, lo que demuestra así que la 4RI puede llegar a intervenir fuertemente incluso en el campo artístico y comunicativo que desde hace décadas marca la pauta para entregar información, entretenimiento e interacción.

Finalmente, en este capítulo se recalca la importancia del cambio de visión social y la necesidad de recibir la virtualidad como una realidad misma; así mismo, se resalta la importancia de la implementación de mecanismos como el big data en la era de la información y la comunicación.

A modo de conclusión, los autores dejan un pliego de recomendaciones en razón a cada uno de los retos abordados en el desarrollo del libro, los cuales pueden ser sintetizados de la siguiente manera: 
- La relevancia de conocer e identificar plenamente la 4RI, para así poder llegar a implementar nuevos mecanismos en el Sistema Nacional de Competitividad, Ciencia, Tecnología e Innovación. De igual manera, se resalta la importancia de establecer parámetros en el uso de tecnologías que podrían afectar la privacidad de los ciudadanos.

- La importancia de la estructuración y aplicación de políticas públicas que fijen metas y planes de trabajo dentro del desarrollo de la implementación de la 4RI.

- El replanteamiento de los sistemas educativos, en todos los niveles, atendiendo así a las nuevas necesidades en el campo laboral.

- El fortalecimiento de mecanismos que garanticen la equidad a las mujeres, las poblaciones afro e indígenas, las víctimas del conflicto armado, entre otros.

- El mejoramiento de los sistemas de recaudo de datos (estadísticos), en general, para así poder generar información verídica sobre los factores que requieren atención prioritaria en aras a la implementación de tecnologías.

- El mejoramiento de los sistemas de vías de acceso a los centros de educación, así como del esfuerzo de las entidades administrativas para equipar y adecuar dichos centros.

- La implementación de mejoras y nuevos sistemas de producción agrícola, para garantizar así la inclusión del campesinado y el desarrollo del sector rural.

- La anticipación del impacto de la sistematización en el plano laboral, para respaldar los derechos laborales y generar mecanismos que hagan frente al desempleo en ciertos sectores, por medio de la capacitación para nuevos empleos.

- La necesidad de entender el principio de seguridad jurídica como un principio que puede adaptarse a los distintos cambios que afloren en el mundo jurídico a raíz de la 4RI; el derecho para mantener su eficacia como regulador de la conducta humana en sus relaciones sociales debe mutar en relación con las circunstancias.

- La implementación de la inteligencia artificial como un medio a través del cual se puede contribuir a la simplificación de los procesos que el legislador colombiano debe surtir para la creación y expedición de normas. 
- La reestructuración de las instituciones jurídicas en Colombia en concordancia con un mundo que avanza de acuerdo con la tecnología, no para la generación de normatividad excesiva, sino para la regulación efectiva de las necesidades. El derecho colombiano necesita estar a la vanguardia del avance tecnológico, y evitar, ante todo, quedarse obsoleto.

- La simplificación del lenguaje reglamentario para superar su construcción ilógica y ambigua que supone un problema a la automatización.

- La construcción de mecanismos y regulaciones que hagan propicia la vigilancia y la protección de los datos personales por parte del Gobierno y las entidades señaladas como competentes para tal fin.

- La articulación de los atributos de la personalidad en el sistema de big data y la generación de escenarios seguros para su salvaguarda y protección.

- La importancia de derribar los mitos que ha generado en la sociedad colombiana la concepción de la tecnología como un mundo análogo; se debe desacelerar la incidencia de las tecnologías en las artes para salvaguardar la esencia de estas que radica en los tiempos y épocas y no en el crecimiento de la tecnología que, por el contrario, suele silenciarlas.

Katty Dayanna Valencia y Wenndy Pedraza 